\title{
The composition of intestinal microbiota and its association with functional constipation of the elderly patients
}

\author{
Mingquan Guo ${ }^{\ddagger 1,2,3}$ (D), Jianfeng Yao ${ }^{4}$, Feng Yang ${ }^{1}$, Wenjian Liu1 ${ }^{1}$, Huijing Bai ${ }^{5}$, Jianxia \\ $\mathrm{Ma}^{4}$, Xingchen Ma ${ }^{1}$, Jinghao Zhang ${ }^{1}$, Yi Fang ${ }^{1}$, Yingxin Miao ${ }^{1}$, Jianqin Sun ${ }^{\ddagger}{ }^{1}$, Yanmei \\ Zhang*,1,2,3 \& Hu Zhao**,1,2,3 \\ ${ }^{1}$ Department of Laboratory Medicine, Huadong Hospital affiliated to Fudan University, Shanghai 200040, PR China \\ ${ }^{2}$ Shanghai Key Laboratory of Clinical Geriatric Medicine affiliated to Shanghai 200040, PR China \\ ${ }^{3}$ Research Center on Aging \& Medicine affiliated to Fudan University, Shanghai 200040, PR China \\ ${ }^{4}$ Department of Gastroenterology, Huadong Hospital affiliated to Fudan University, Shanghai 200040, PR China \\ ${ }^{5}$ Clinical Nutrition Center, Huadong Hospital affiliated to Fudan University, Shanghai 200040, PR China \\ *Author for correspondence: 15618653286@163.com \\ **Author for correspondence: hubertzhao@163.com \\ $\ddagger$ Authors contributed equally
}

\begin{abstract}
Aim: To identify intestinal microbiota compositions in elderly functional constipation (FC) patients. Materials \& methods: Fecal samples from $61 \mathrm{FC}$ patients and 48 healthy age-matched volunteers were analyzed through 16S rRNA gene sequencing. Results: The intestinal microbiota compositions of FC patients were significantly different from healthy controls. Additionally, the species diversity of healthy controls was greater than that of FC patients. Indeed, the abundance of Firmicutes and Proteobacteria was significantly decreased, whereas that of Bacteroides, Prevotella, Lactococcus, Ruminococcus and Butyricimonas was remarkably increased in FC patients. Conclusion: Elderly FC patients appear to have a unique intestinal microbiota profile. Our findings should provide insight regarding the pathogenic mechanism of FC and evidence for exploring new therapeutic strategies in elderly FC patients.
\end{abstract}

First draft submitted: 9 October 2019; Accepted for publication: 27 January 2020; Published online: 21 February 2020

Keywords: 16 s rRNA gene sequencing • functional constipation $\bullet$ intestinal microbiota composition $\bullet$ the elderly

Functional constipation (FC), otherwise known as chronic idiopathic constipation (CIC), is a very common pathological condition [1,2]. As a symptom-based gastrointestinal disorder without organic abnormalities, FC has an average global prevalence of approximately $14 \%$, ranging from 1.9 to $40.1 \%$, depending on the population [35]. In China, approximately $10-14.9 \%$ of the population suffers from chronic constipation and the condition is particularly prevalent among the elderly, leading to serious impacts on quality of life [6-8]. To date, China is considered to have the highest ratio of elderly people in the world, with the total number of elderly people aged 60 years or greater having reached 212 million in 2014, accounting for $15.5 \%$ of the total population [9-11]. This aging population has caused an increase in the incidence of geriatric diseases, with FC reported as an important risk factor for various diseases, including cardiovascular and cerebrovascular diseases, colon cancer and even death [12].

An extremely dense and diverse bacterial ecosystem resides in the human gastrointestinal tract, which coevolves as an integral part of human biology [13]. Recent studies have indicated that intestinal microbiota play a vital role in human health and is related to various physiological activities of the host. Indeed, alterations in intestinal microbiota have been confirmed closely associated with various diseases, such as inflammatory bowel diseases (IBDs), allergy, obesity, malnutrition, neurological disorders, Type 2 diabetes and cancer [14-17]. While the pathophysiological mechanisms of FC are not fully understood, the brain-gut bacteria axis (BGBA) is a novel concept in gastroenterology that may be used to elucidate the pathogenesis and clinical manifestations of FC $[18,19]$. Indeed, several studies have shown that profiles of fecal microbiota are different between constipated patients and healthy people $[20,21]$. However, these studies primarily have involved children and adult patients, thus limited 
information exists for elderly patients. Considering the increasing global elderly population and the severity of FC, a greater understanding of the pathogenesis underlying this condition is necessary in order to improve the health status of elderly FC patients [22,23].

In this study, for better understanding the relationship between intestinal microbiota composition and FC in the elderly, we compared the intestinal microbiota compositions and diversity between elderly patients with FC and healthy controls. Our findings should provide additional insight into the pathophysiological mechanisms underlying FC and evidence to support relevant microbiota treatment for elderly FC patients.

\section{Materials \& methods}

\section{Patient cohorts}

This study was conducted at Huadong Hospital in Shanghai, China. Patients over 60 years old diagnosed with FC were recruited for the study from January to December 2018. Data and samples were collected from the participants from June to December 2018. The inclusion criteria were as follows: patients had been diagnosed with FC in accordance with the Rome III criteria from the American College of Gastroenterology (ACG); patients had no diseases of the gastrointestinal tract or other metabolic diseases but had a history of constipation within the past 5 years. Patients were excluded if the following criteria were met: the constipation was secondary to other diseases (e.g., severe liver dysfunction, metabolic conditions, malignant tumors, renal dysfunction, endocrine conditions) or intervention (e.g., drugs) in the past 5 years; patients had been diagnosed with a psychiatric disorder; patients had taken antibiotics, probiotics or bismuth agents during the first 3 months of the study. After informed consent, eligible patients were enrolled in a 2-week observation period and the constipation severity was determined by Agachan-Wexner score. Based on this score, FC patients were divided into two groups, namely, mild FC representing a light syndrome (FCL group) and severe FC representing a heavy syndrome (FCH group). In our study, information from each participant could be timely identified and understood during or after data collection. This study was performed according to the human subject protocols approved by the Ethics Committee (Ethics Committee Program No. 2018k045) of Huadong Hospital.

\section{Sample collection \& DNA extraction}

Fecal samples (about $5 \mathrm{~g}$ of feces per person) were collected from 61 patients and 48 healthy volunteers. Samples were frozen at $-80^{\circ} \mathrm{C}$ within $2 \mathrm{~h}$, without the addition of any stabilization reagent, until further analysis. Bacterial genomic DNA was extracted using the Fast DNA SPIN extraction kit (MP Biomedicals, CA, USA) according to the manufacturer's instructions. The extraction quality of the DNA was determined by its concentration and purity. The concentration was confirmed to be at least $5 \mathrm{ng} / \mu \mathrm{l}$ and the purity was validated by OD260/280 readings of 1.8-2.0 by NanoDrop ND-1000 spectrophotometer (Thermo Fisher Scientific, MA, USA).

\section{Amplicon sequencing of $16 \mathrm{~S}$ rRNA gene}

The bacterial 16S rRNA gene V3-V4 region was used for PCR amplification. The primers used were as follows: 338F (5'-ACTCCTACGGGAGGCAGCA-3'); reverse primer 806R (5'-GGACTACHVGGGTWTCTAAT-3').

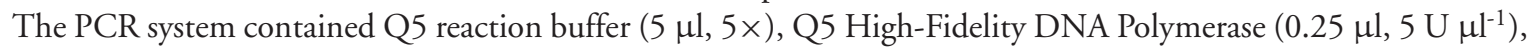

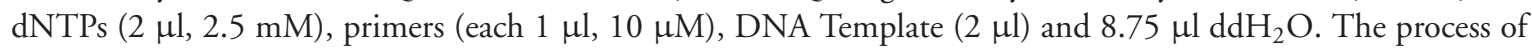
amplification included initial denaturation at $95^{\circ} \mathrm{C}$ for $5 \mathrm{~min}, 28$ cycles consisting of $15 \mathrm{~s}$ denaturation at $95^{\circ} \mathrm{C}$, $30 \mathrm{~s}$ annealing at $55^{\circ} \mathrm{C}$ and $30 \mathrm{~s}$ extension at $72^{\circ} \mathrm{C}$, with a final extension at $72^{\circ} \mathrm{C}$ for $10 \mathrm{~min}$. The negative controls were conducted with $\mathrm{ddH}_{2} \mathrm{O}$. Agencourt AMPure Beads (Beckman Coulter, IN, USA) were employed for the purification of PCR amplicons. The PicoGreen dsDNA Assay Kit (Invitrogen, CA, USA) was used to quantify the concentrations. Amplicons were maintained in equal amounts, and pair-end $2 \times 300$ bp was sequenced by the Illumina MiSeq platform in conjunction with the MiSeq Reagent Kit v3 at Shanghai Personal Biotechnology Co., Ltd (Shanghai, China).

\section{Bioinformatics analysis}

The Quantitative Insights Into Microbial Ecology (QIIME, v1.8.0) pipeline was used to process sequencing data as described previously [24]. In brief, raw sequencing reads with exact matches to the barcodes were assigned to respective samples and identified as valid sequences. Screening criteria for low-quality sequences included: sequences with a length $<150 \mathrm{bp}$, a Phred mean fraction $<20$, sequences with unclear bases and single nucleotide repeats $>8$ bp [25,26]. After chimera detection, the remaining high-quality sequences were clustered by $97 \%$ sequence 
Table 1. Demographics information of functional constipation patients and healthy controls.

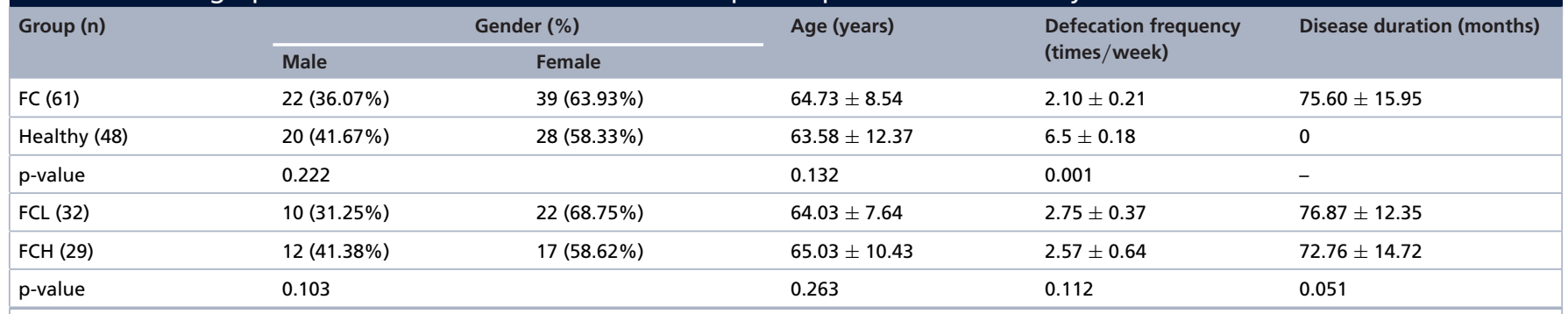

FC: Functional constipation; FCH: Severe FC representing a heavy syndrome; FCL: Mild FC representing a light syndrome.

consistency through UCLUST as an operational taxon (OTUs) [25]. OTU taxonomic classification was performed by searching through BLAST for representative sequences in the Greengenes Database using the best hit [27]. A OTU table was generated further to show the OTU abundance of each sample and the taxonomy. The OTUs less than $0.001 \%$ of the total sequences across all samples were deleted. In order to minimize the difference in sequencing depth between the different samples, we generated an average, round and refined OTU table for further analysis by averaging 100 OTU subsets of uniform resamples at the minimum sequencing depth of $90 \%$.

OTU-level alpha diversity indices, like the ACE metric (Abundance-based Coverage Estimator), Chao1 richness estimator, Simpson index and Shannon diversity index were calculated through OTU table in QIIME. OTU-level ranked abundance curves were generated to compare the richness and evenness of the OTUs among the samples. Beta diversity analysis was performed to investigate the structural variation of microbial communities across the samples using UniFrac measures and was visualized by principal coordinate analysis (PCoA) [28,29]. Metastases visualized as violin plots was used to statistically compare the taxa abundances at the phylum, class, order, family, genus and species levels among the samples or groups [30,31].

\section{Statistical analysis}

Statistical analysis was performed by Statistica software version 10.0 (StatSoft Inc., Tulsa, OK, USA). Continuous variables were expressed as mean \pm standard deviation and the differences were compared by one-way ANOVA. Nonparametric t-tests and linear discriminate analysis effect size (LefSe) were used in our study. p-values $<0.05$ were considered to be statistically significant.

\section{Results}

\section{Patient demographics}

Demographic information from the study subjects was shown in Table 1. In total, 61 patients in the FC group and 48 healthy/normal individuals in the healthy control group met the eligibility criteria of this study. Among them, 39 female and 22 male patients were in the FC group, and 28 female and 20 male subjects were in the healthy control group. The mean age was 64.73 years old for the FC group and 63.58 years old for the healthy control group. The duration of constipation was $75.60 \pm 15.95$ months for the FC group and the mean defecation frequency was $2.10 \pm 0.21$ /week in the FC group and $6.5 \pm 0.18 /$ week $(\mathrm{p}=0.001)$ in the healthy group. According to the Agachen-Werner score, the 61 FC patients were further divided into two subgroups, with 24 in the FCL group and 37 in the FCH group.

\section{Evaluation of the alpha diversity in FC patients \& healthy controls}

In order to analyze the species diversity and evenness between the FC group and healthy controls, we estimated the community species richness using the abundance-based coverage estimator (ACE) index. All the sequenced read pools showed a tendency to reach a plateau. In all cases, so the retrieved sequencing data were considered sufficient to cover most of the biodiversity contained in the sample. The ACE indexes of FC patients and healthy controls were significantly different $(936.03 \pm 250.91$ vs $877.39 \pm 322.79$, respectively; $\mathrm{p}=0.001)$.

\section{Taxonomic profiling of the intestinal microbiota of FC patients \& healthy controls}

A total of 4,586,518 sequence datasets were obtained by Illumina MiSeq from 109 samples, and these were used to establish gut bacterial diversity. The overall microbial composition of each group at the phylum level is shown 


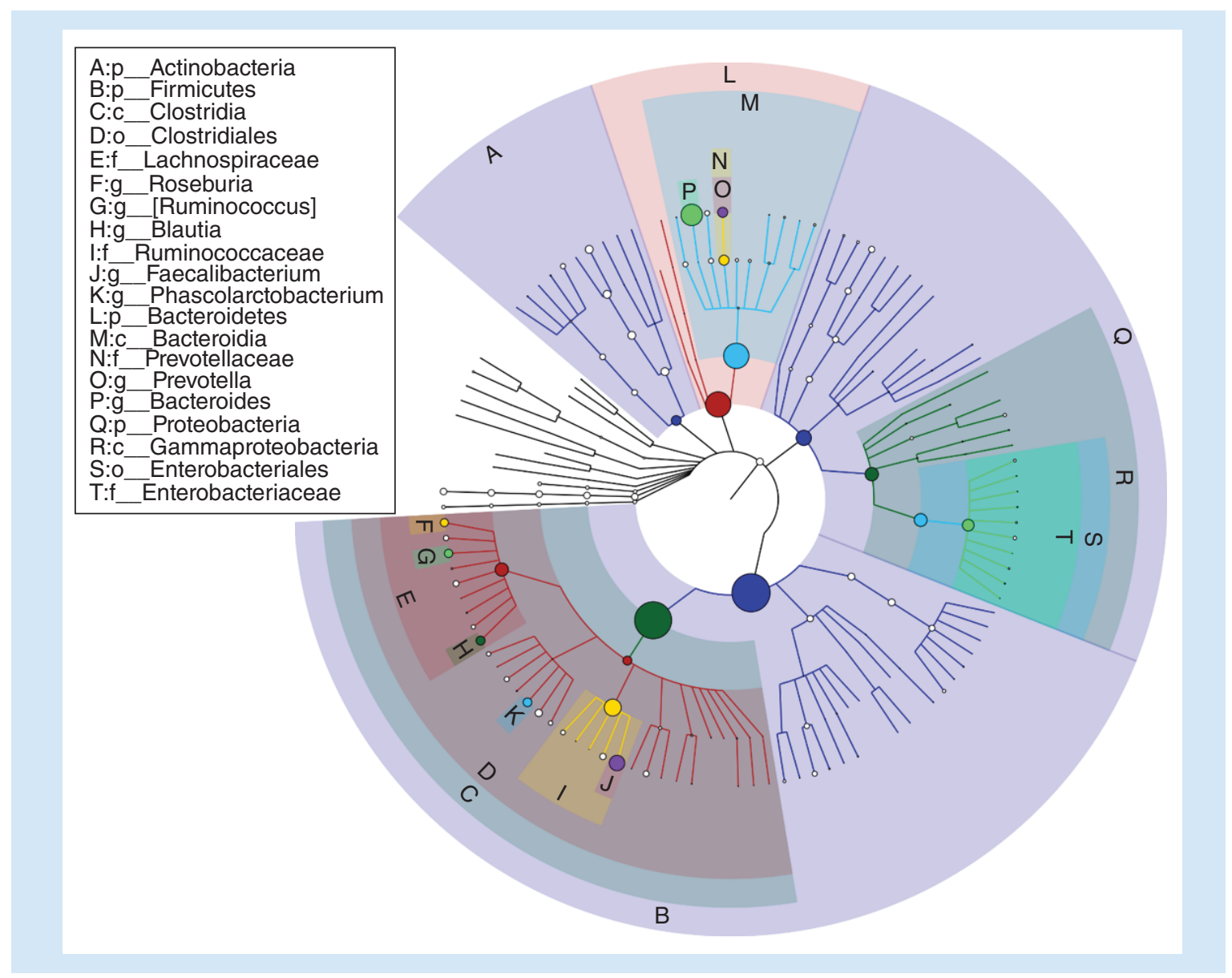

Figure 1. The overall intestinal microbiota composition of all subjects (61 functional constipation patients and 48 healthy controls) at the phylum level.

in Figure 1. A total of 18 different bacterial phyla were identified in all samples. The majority of the sequences belonged to Firmicutes (65.42\%), Bacteroidetes (16.14\%), Proteobacteria (13.43\%) and Verrucomicrobia (3.23\%), which encompassed more than $98 \%$ of all sequences. The sequences were then classified into 170 different genera. Ten genera, including Bacteroides, Parasporobacterium, Veillonella, Streptococcus, Natronoflexus, Psendoflavonifractor, Isobaculum, Alkalitalea, Sporotomaculum and Cosenzaea, were found to account for more than $1 \%$ of the sequences.

Differences of bacterial community between FC patients \& healthy controls at the phylum level According to the taxonomic results, the overall microbial composition was then compared between the groups (Figure 2). Bacterial phyla with a relative abundance of $>0.1 \%$ were analyzed. Firmicutes was the most common phylum in both groups, representing $65.39 \%$ and $55.86 \%$ in the healthy and FC groups, respectively. Bacteroidetes was the second dominant phylum, accounting for 13.70 and $33.19 \%$ in the healthy and FC groups, respectively $(\mathrm{p}=0.001)$. Proteobacteria constituted the third most abundant phylum $(13.33$ vs $6.08 \% ; \mathrm{p}=0.006)$ present in the healthy and FC groups respectively. Actinobacteria ( $4.33 \%$ vs $0.314 \% ; \mathrm{p}=0.002)$ and Verrucomicrobia (3.76 vs $1.94 \% ; \mathrm{p}=0.031$ ) were also more abundant in the healthy group compared with the FC group (Figure $2 \mathrm{~A}$ ). When the overall microbial composition was analyzed among the three groups (healthy, FCL and FCH) on the phylum level, histogram analysis showed that there were significant differences in the species abundance of some phyla. Bacteroidetes increased in the serious constipation patients (FCH), whereas Firmicutes, Proteobacteria and Actinobacteria were decreased in these patients. Bacteroidetes accounted for $16.06 \%$ of the abundance in the healthy group, $31.43 \%$ in the FCL group and $35.18 \%$ in the FCH group. Firmicutes accounted for $61.43 \%$ in the healthy group, $52.56 \%$ in the FCL group and $58.74 \%$ in the FCH group, respectively. Proteobacteria accounted for $13.36 \%$ 

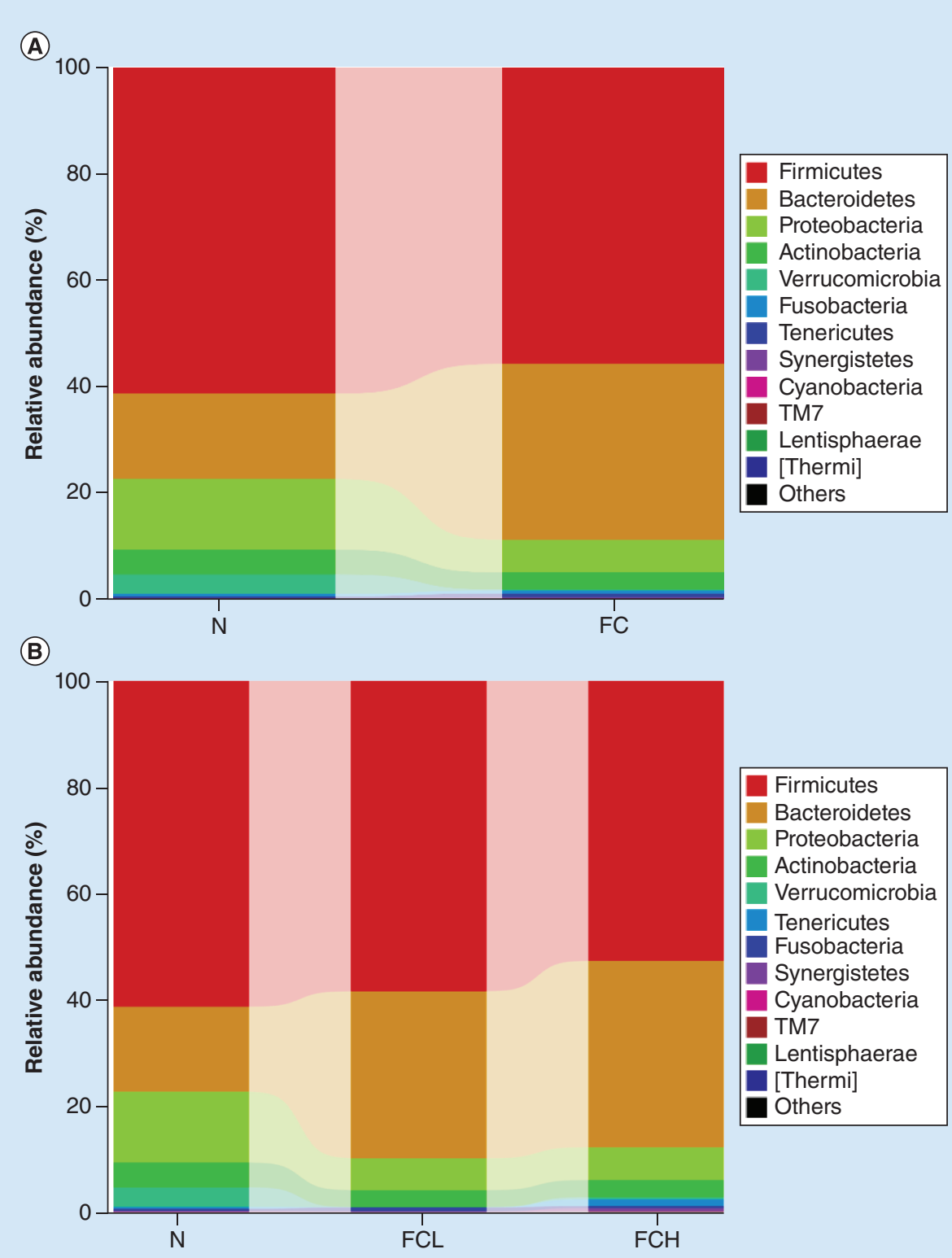

Figure 2. The overall intestinal microbiota composition between functional constipation patients and healthy controls at the phylum level. (A) The dominant phyla in healthy and FC groups, (B) The dominant phyla in healthy, $\mathrm{FCL}$ and $\mathrm{FCH}$ groups.

FC: Functional constipation; FCH: Severe FC representing a heavy syndrome; FCL: Mild FC representing a light syndrome; N: Healthy group.

in the healthy group, $6.04 \%$ in the FCL group and $6.21 \%$ in the FCH group. Actinobacteria accounted for $4.65 \%$ in the healthy group, $3.15 \%$ in the FCL group and $3.20 \%$ in the FCH group (Figure 2B).

\section{The bacterial community between FC patients \& healthy controls at the genus level}

There were 334 bacterial taxa obtained from genus-level analysis (Figure 3) and we found that the microbial composition differed significantly between the FC and healthy groups. Bacterial genera at a relative abundance of $>0.1 \%$ were analyzed and the compositions were as follows: Bacteroides (12.51 vs 23.89\%), Faecalibacterium (9.43 vs 9.44\%), Prevotella (1.23 vs 6.29\%), Akkermansia (3.68 vs $0.19 \%)$, Blautia (3.54 vs $2.93 \%)$, Veillonella (1.33 vs $0.23 \%)$, Ruminococcus (1.89 vs 3.37\%) and Streptococcus (2.82 vs $0.09 \%$; Figure 3A). Our analysis at the genus level revealed that some species, including Prevotella, Akkermananisia, Lactococcus, Atopobium, Thermus, 


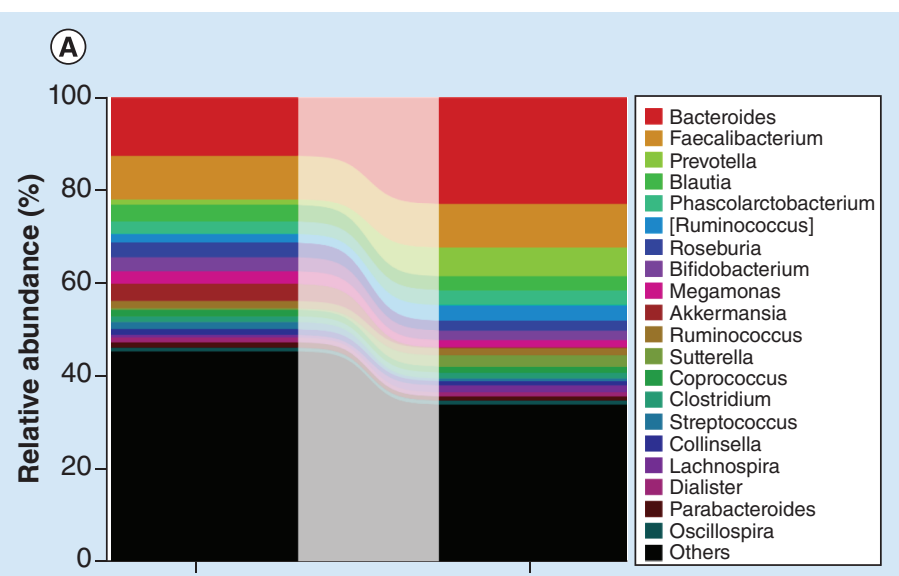

H

(B)

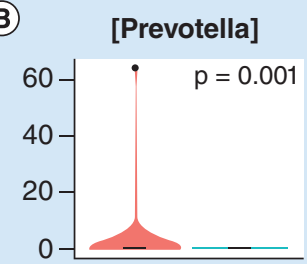

Butyricimonas
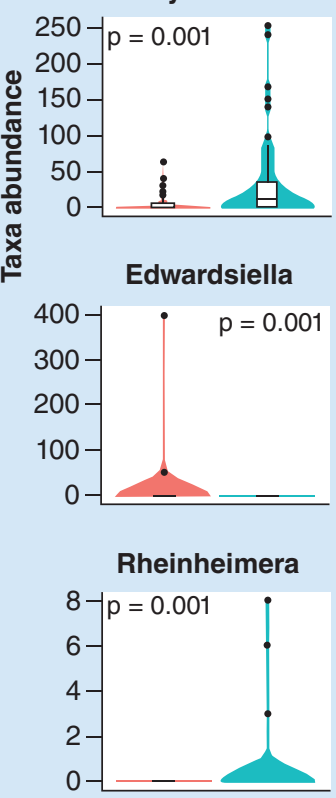

FC
Akkermansia
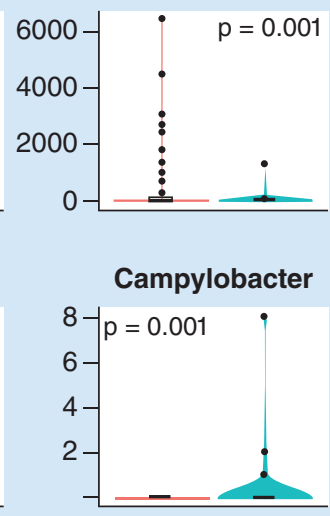

Epulopiscium
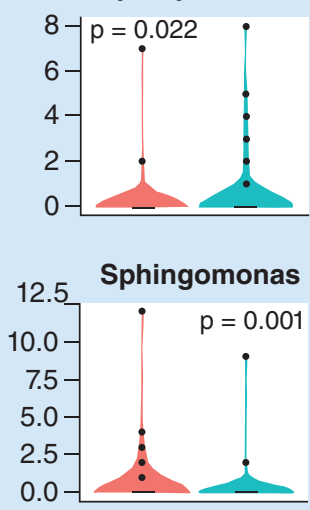

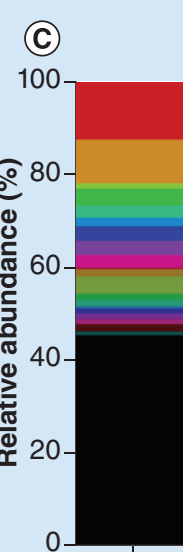

$\mathrm{H}$

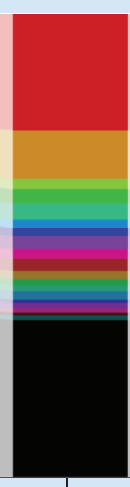

FCL
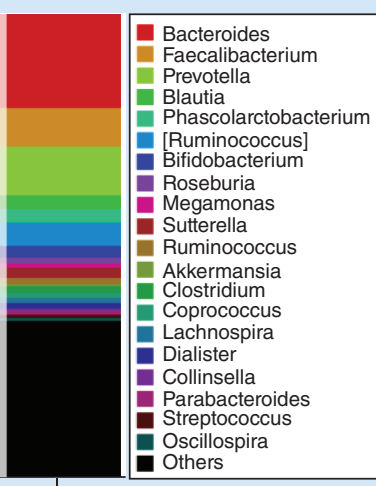

$\mathrm{FCH}$
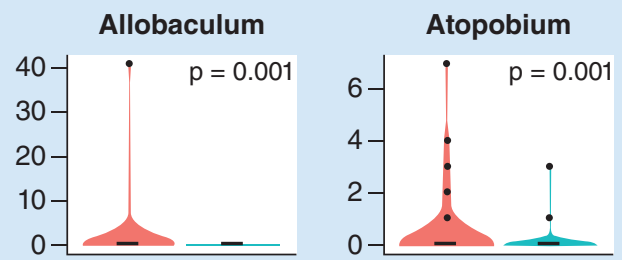

Bacteroides

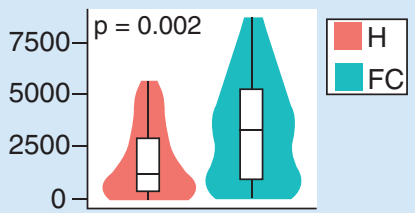

Clostridium

Dehalobacterium

Deinococcus
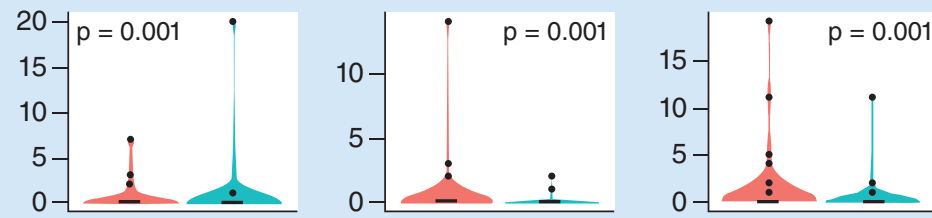

Pseudomonas

Escherichia
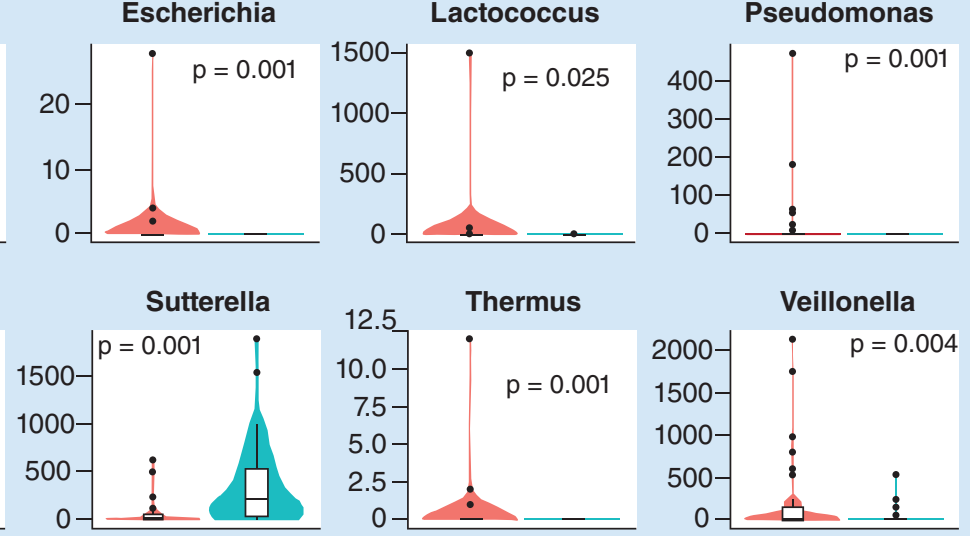

Group

Figure 3. The intestinal microbiota composition between functional constipation patients and healthy controls at the genus level. (A) The dominant genera in healthy and FC groups, (B) Top 20 genera with significant differences at the genus level, (C) The dominant genera in healthy, $\mathrm{FCL}$ and $\mathrm{FCH}$ groups.

FC: Functional constipation; FCH: Severe FC representing a heavy syndrome; FCL: Mild FC representing a light syndrome; H: Healthy group.

Dehalobacterium and Vellonella, were significantly more abundant in the healthy group compared with the FC group. However, Bacteroides, Butyricimonas, Sutterella, Clostridrium and Rheinheimera were significantly less abundant in the healthy group compared with the FC group (Figure 3B). The overall microbial compositions were then analyzed among the three groups (healthy, FCL and FCH) at the genus level (Figure 3C). Bacteroides accounted for $12.36 \%$ in the healthy group, $20.34 \%$ in the FCL group and $23.57 \%$ in the FCH group, respectively. Prevotella accounted 
for $1.17 \%$ in the healthy group, $2.25 \%$ in the FCL group and $10.54 \%$ in the FCH group. Blautia accounted for $4.65,3.31$ and $2.87 \%$ in the healthy, FCL and FCH groups, respectively. Ruminococcus accounted for $2.06 \%$ in the healthy group, $2.43 \%$ in the FCL group and $6.17 \%$ in the FCH group. Significant differences were observed among the three groups at the genera level for Atopobium, Escherichia, Rheinheimera, Veillonella, Clostridium, Prevotella, Thermus, Deinococcus, Dehalobacterium, Sutterella, Psuedomonas and Phylibacterium.

\section{Difference in the relative abundance between FC patients \& healthy controls}

To evaluate the differences in microbiota composition, statistical analysis was performed to compare the average relative abundance in the different genera groups with an absolute percentage difference $>0.1 \%$. Differentially abundant genera species with significant differences between the two groups were then identified. We confirmed greater species numbers in the healthy group compared with the FC group (Figure 4). Interestingly, the comparison between the FC and healthy groups datasets showed that the profiles obtained from the healthy controls were characterized by a statistically significant over-representation of Gammaproteobacteria (absolute 5.09\%; $\mathrm{p}=0.0002$ ), Enterobacteriaceae (absolute 5.06\%; $\mathrm{p}<0.0001$ ), Verrucomicrobia (absolute 4.56\%; $\mathrm{p}=0.003$ ), Alcaligenaceae (absolute 4.38\%; p = 0.001), Enterobacteriaceae (absolute 4.05\%; p < 0.0001), Akkermansia (absolute 4.56\%; $\mathrm{p}=$ 0.003), Lactobacillus (absolute 3.83\%; $\mathrm{p}=0.005$ ), Coriobacteriaceae (absolute 4.20\%; $\mathrm{p}=0.03$ ) and Actinomycetaceae (absolute 2.31\%; $\mathrm{p}=0.002$ ). In contrast, several taxa, including Bacteroidales (absolute 5.52\%; $\mathrm{p}<0.0001$ ), Butyricimonas (absolute 3.36\%; p = 0.001), Faecalibacterium (absolute 3.54\%; $<<0.0001$ ), Alcaligenaceae (absolute 4.38\%; $\mathrm{p}<0.0001$ ), Alcaligenaceae (absolute 4.38\%; $\mathrm{p}<0.0001$ ), Burkholderiales (absolute 4.38\%; $\mathrm{p}<0.0001$ ), Lachnospira (absolute 4.17\%; $<<0.0001$ ) and Lentisphaeria (absolute 2.53\%; $\mathrm{p}=0.021$ ) were decreased, indicating they may be a factor in the dysbiosis of the microbiota in FC patients.

\section{PCoA analysis of the samples}

To further evaluate the inter-individual differences between the FC and healthy groups, we assessed and represented the results through PCoA analysis (Figure 5). Notably, the resulting PCoA plot showed that the samples were primarily grouped as two different clusters, corresponding to either the FC patients or healthy controls. In addition, we found that corresponding samples selected by their microbiota profiles were shown closer to the average for each group. Furthermore, our PCoA results revealed a significant separation in the bacterial community composition between the FC patients and healthy controls by the first two principal component scores of PC1 and PC2 (11.38 and 25.60\%) of the explained variance, respectively.

\section{Discussion}

In recent years, chronic FC micro-ecological studies have garnered increased attention [32,33]. The symptoms caused by FC, including anxiety, insomnia, dizziness, stomach ache and fatigue are relatively common in clinical practice, particularly in the elderly $[6,34]$. However, despite FC being a common and burdensome gastrointestinal disease, its etiology and pathophysiology remain unclear $[35,36]$. Growing evidence has indicated that chronic FC may be related to physiological functions, such as intestinal dynamics, but also intestinal micro-ecological changes [37,38]. Therefore, it is crucial to elucidate the distribution characteristics of intestinal bacteria in the elderly with FC.

In humans, establishing and maintaining a beneficial microbiome balance is necessary for the normal functioning of the intestines [39,40]. Several studies have reported that obesity, diabetes, C. diffcile infection, IBD, irritable bowel syndrome (IBS) and various chronic diseases present with decreased fecal microbiota diversity [41-44]. In the current study, we found that the intestinal microbiota diversity of the FC group was significantly lower than that of the healthy group. In addition, we found that the abundance of the genus Lactococcus and Prevotella in the FC group was significantly lower than that in the healthy group. Similarly, a decline of this genus in the intestinal microbiota has also been found in both children and adults with constipation, suggesting that the loss of Lactococcus and Prevotella may play a key role in the development of constipation at different ages $[23,37]$. Furthermore, the abundance of Clostridium was significantly lower in healthy controls. Previous findings have indicated that Clostridium is increased in children and adults with constipation [45,46]. In addition, some bacteria species presented a significant difference in the elderly with FC, including Epulopiscium, Lachnospira, Thermos and Serratia, which have not been previously reported, indicating a possible specificity in the intestinal microbiota profiles of elderly FC patients.

The present study revealed that the phylum Bacteroidetes was present at about two-times greater levels in the microbiota of the FC group compared with the healthy group. In addition, the microbiota composition of the severe FCH group was significantly more abundant in Bacteroidetes than that of the FCL patients, indicating Bacteroidetes 


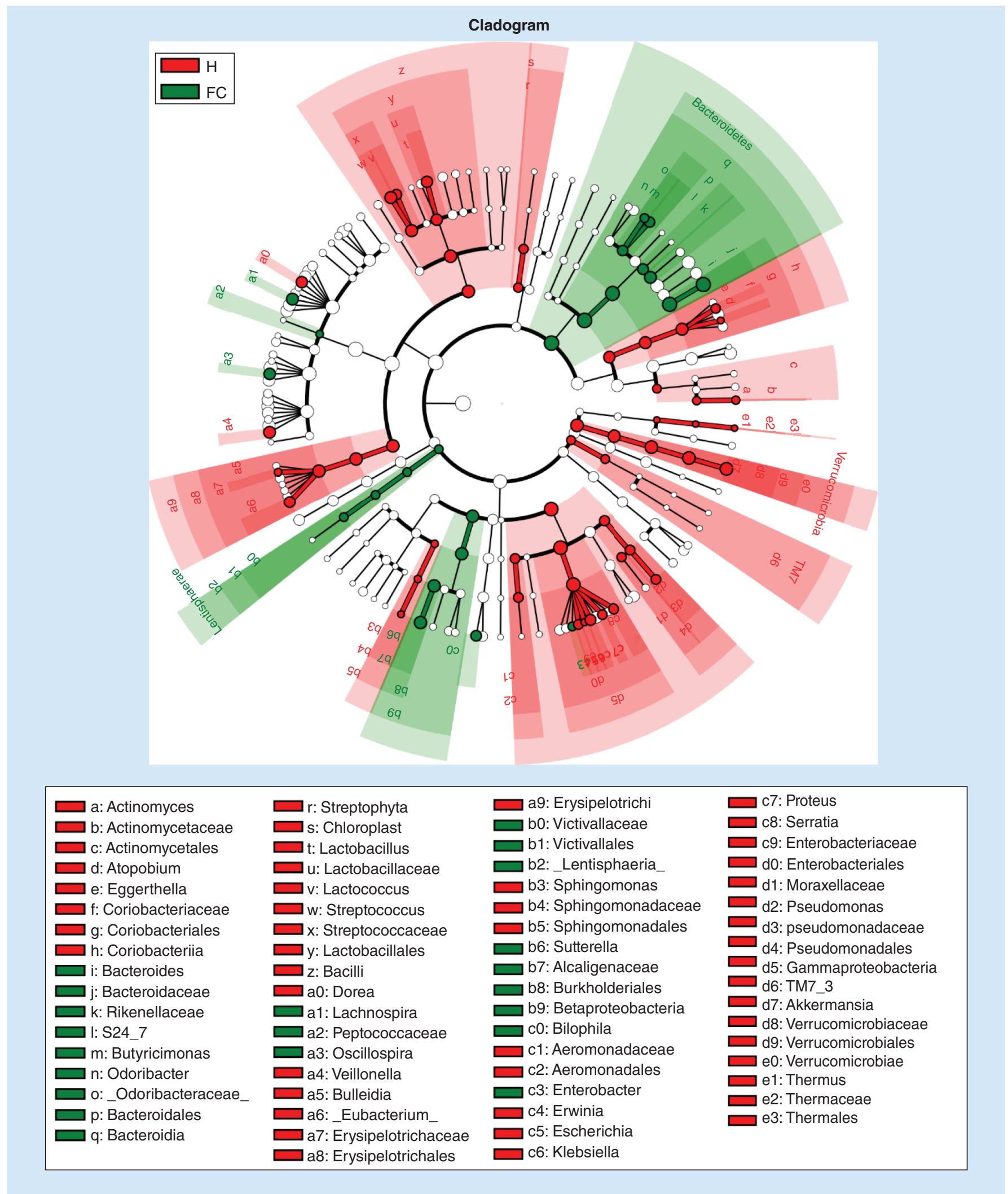

Figure 4. The comparison of different intestinal microbiota species between functional constipation patients and healthy controls based on the classification level tree. The classification level tree shows the hierarchical relationship of all taxonomic units in the samples from the phylum to the genus level (arranged in order from the inner circle to the outer circle). The node size corresponds to the relative abundance of taxonomic units. The green nodes represent species with significantly higher abundance in functional constipation group compared with healthy group. The red nodes represent species with significantly higher abundance in healthy group compared with functional constipation group.

FC: Functional constipation; $\mathrm{H}$ : Healthy group. 


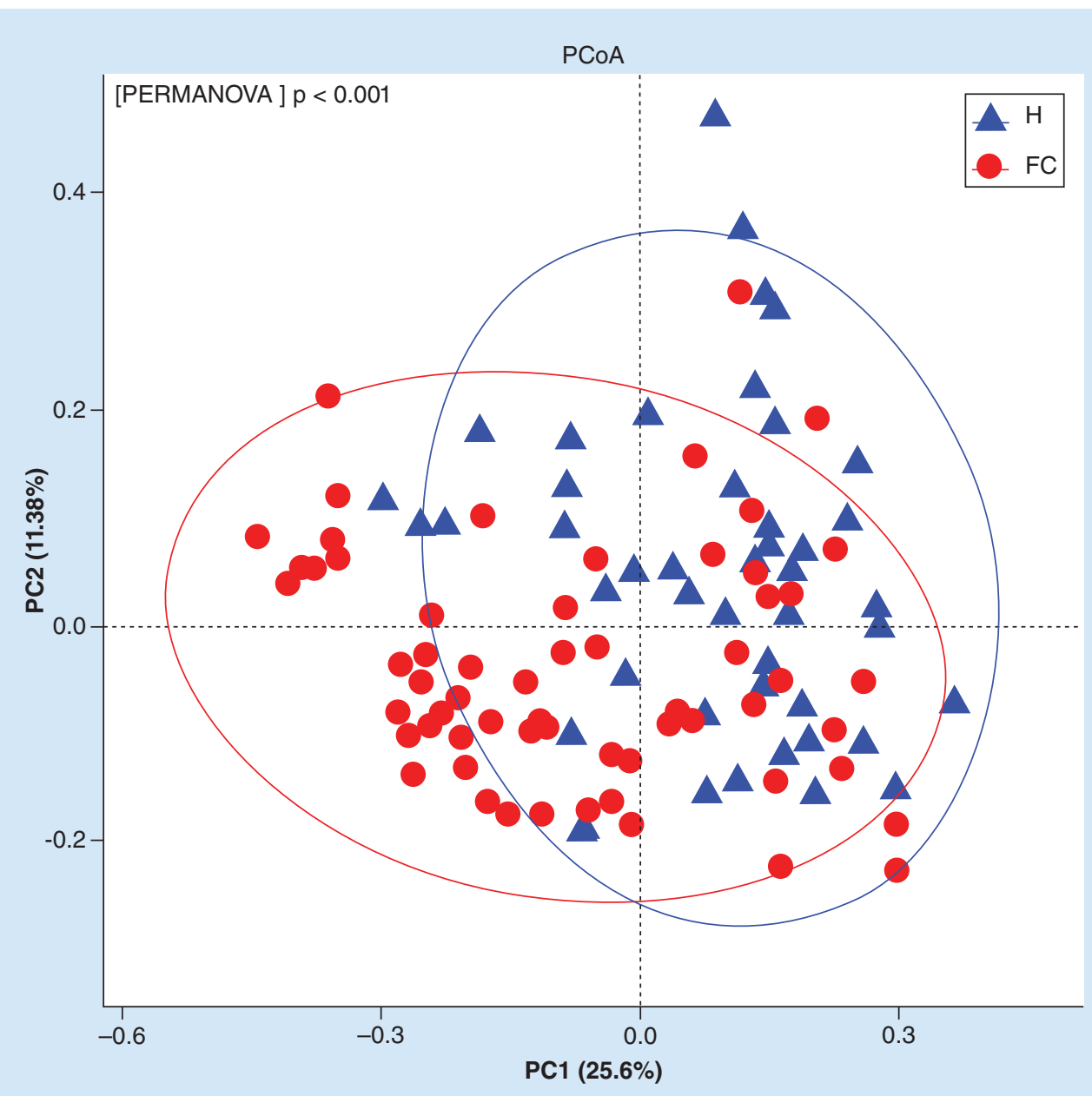

Figure 5. The plot of unweighted UniFrac principal coordinates analysis based on the relative abundance of operational taxon units. Each symbol represents a group, the blue triangles represent the samples of healthy group and the red circles represent the samples of functional constipation group.

FC: Functional constipation; H: Healthy group; PC1: Principal coordinate 1; PC2: Principal coordinate 2; PCoA: Principal coordinates analysis.

may also be positively related with the severity of FC. In support of our findings, Lovell reported the positive association of Bacteroidetes with methylglyoxal degradation pathways and Wu et al. demonstrated that Bacteroidetes in feces is negatively associated with dietary fiber intake $[47,48]$. The results of our study demonstrated a negative relationship between Firmicutes, Proteobacteria and Actinobacteria and FC. Consistent with our findings, Kang et al. showed that the relative abundance of Proteobacteria is significantly decreased in constipated patients, and the Firmicutes phylum has been reported to be positively associated with the pathways involved in hydrogen production and methanogenesis, which may indicate a potential mechanism in FC development [49-51]. Collectively, the results of these studies suggest that changes in some microbiota may affect FC and its related symptoms.

In addition, we found a distinct difference between the healthy and FC groups at the genus level. Bacteroides, Ruminococcus, Lachnospiraceae, Prevotella and others were more abundant in the FC group, but Akkermansia and Veillonella were found to be less abundant. Based on the Agachan-wexner score, we found that Butyricimonas, Porphyromonadaceae and Thermus demonstrated an increasing trend in the healthy, FCL and FCH groups, but Rheinheimera and Lactobacillus were the opposite. Similarly, Zhu et al. reported the significant increase of Prevotella in constipated patients [20]. In a study of the intestinal microbiota in people with autism, Ruminococcus was also found to be negatively related with constipation symptoms [52]. In addition, genera such as Ruminococcus, Prevotella and Bacteroides have been found to be directly and inversely related with colonic transit, which has been suggested 
for IBS [53]. These findings indicate that Bacteroides, Ruminococcus and Prevotella may be positively related to FC in the elderly and Butyricimonas, Porphyromonadaceae and Thermus may be related with disease occurrence and development.

As a member of a versatile class of microbial-produced metabolites, butyrate has been shown to be metabolized by microbiota in the intestine [54]. In our study, we found that Butyricicoccus, Campylobacter and Faecalibacterium in the FC group were significantly higher than in the healthy group. Therefore, it is possible that these butyrate-producing bacteria change the metabolism of the host, thus affecting gastrointestinal motility. This hypothesis is supported by animal experiments that have demonstrated that butyric acid may inhibit colonic smooth muscle contraction and cause slow transit constipation, in conjunction with the colonic absorption of water, which makes the feces dry and hard [55,56]. Interestingly, children with constipation have been identified to have an increased abundance of Clostridium spp, Ruminococcus [37] and adults (mean age $=50.8 \pm 15.7$ years) with constipation have been shown to have an increased abundance of Flavobacterium and Eubacterium [51]. These results have indicated that bacteria such as Butyricicoccus, Clostridium, Ruminococcus, Flavobacterium, Eubacterium and other butyric acid-producing bacteria may be key microbiota for the development of FC.

Although the reported effect of probiotics on treating chronic constipation still remains inconsistent, many studies have confirmed that probiotics have a positive effect on chronic constipation [57,58]. For example, the studies showed that probiotics and fibers might efficiently affect bowel diseases, such as functional bowel disorders, IBS and FC by altering the gut microbial environment [59,60]. A study about probiotic interventions including 21 randomized controlled trials showed that probiotic therapy improved the overall symptom response and life quality for IBS patients compared with placebo [61]. The inconsistent effects of probiotics in different studies may be resulted from several factors, such as ages, living conditions and lifestyles. Therefore, our study illuminated the distribution of intestinal microbiota in elderly Chinese FC patients, which would lay the foundation for revealing the pathogenic mechanism of FC and provide evidence for exploring effective therapy strategy.

\title{
Conclusion
}

Microbes and the host response affect the relationship between health and disease, and gut microbes are known to play an important role in host physiology, health and disease. In this study, we evaluated microbial community compositions in an elderly FC population. The key findings of our study were: the fecal microbiota profiles from elderly patients with FC significantly differed from those of healthy subjects; the abundance of Bacteroidetes in the FC group was increased, whereas that of Firmicutes and Proteobacteria was decreased, compared with healthy subjects; elderly patients with FC had a unique intestinal microbiota profile compared with previous reports of children and adult FC patients; and there was a relationship between the distribution characteristics of the microbiota and the severity of FC. These findings provide additional insight into the mechanisms underlying FC and evidence for the development of novel interventions for elderly FC patients.

\section{Summary points}

- This study focused on the intestinal microbiota compositions and the presence of key species in elderly functional constipation (FC) patients.

- The abundance of Firmicutes and Proteobacteri was significantly reduced, while that of Bacteroides, Prevotella, Lactococcus, Ruminococcus and Butyricimonas were increased, in elderly FC patients.

- The intestinal species diversity in healthy people was greater than elderly FC patients.

- The intestinal microbiota compositions of elderly FC patients were related to disease severity.

\begin{abstract}
Authors' contributions
M Guo and J Yao composed the manuscript and analyzed the data; F Yang, H Bai, W Liu, X Ma, J Ma, Y Fang, J Zhang, Y Miao provided the samples and collected the clinical data; J Sun, $\mathrm{H}$ Zhao and $Y$ Zhang designed and coordinated the study. All the authors approved the final manuscript.
\end{abstract}

Acknowledgments

We would like to thank Z Xue from the Shanghai Personal Biotechnology Co., LTD, for her help with Bioinformatics and statistical analysis. 


\section{Financial \& competing interests disclosure}

This work was supported by the 'Medical guidance support project' of Shanghai Science and Technology Committee (grant no.18411960600), Shanghai Shenkang Hospital Development Center 'New frontier technology joint research project' (grant no. SHDC12015107), Subproject of the national high technology research and development program '863 project' (grant no. 2015AA021107-019), Shanghai Science and Technology Committee 'Lead project' (grant no.16411968000), National Natural Science Foundation Grant of China (grant no.81602072). Funding was also received from Shanghai 'Rising Stars of Medical Talent' Youth Development Program, Outstanding Youth Medical Talents of 2018, Shanghai Science and Technology Committee 'Scientific and technological innovation action projects' (grant no.18411950800), Shanghai health and family planning commission youth project of scientific research subject (grant no. 20184Y0032). The authors have no other relevant affiliations or financial involvement with any organization or entity with a financial interest in or financial conflict with the subject matter or materials discussed in the manuscript apart from those disclosed.

Medical writing support was provided by Enago (http://futuremedicine.enago.com) for pre-submission editing service and was funded by Shanghai Shenkang Hospital Development Center 'New frontier technology joint research project' '(grant no.: SHDC 12015107).'

\section{Ethical conduct of research}

This study was performed in accordance with human subject protocols approved by the Ethics Committee (ethics approval number: 2018D007) of Huadong Hospital affiliated to Fudan University.

\section{Open access}

This work is licensed under the Attribution-NonCommercial-NoDerivatives 4.0 Unported License. To view a copy of this license, visit http://creativecommons.org/licenses/by-nc-nd/4.0/

\section{References}

1. Lee KN. The clinical implications of overlap between constipation and common functional gastrointestinal disorders. $J$. Neurogastroenterol. Motil. 23(4), 485-486 (2017).

2. Vieira MC, Negrelle IC, Webber KU, Gosdal M, Truppel SK, Kusma SZ. Pediatrician's knowledge on the approach of functional constipation. Rev. Paul. Pediatr. 34(4), 425-431 (2016).

3. Ortiz V, Garcia-Campos M, Saez-Gonzalez E, Delpozo P, Garrigues V. A concise review of opioid-induced esophageal dysfunction: is this a new clinical entity? Dis. Esophagus 31(5), (2018).

4. Zhang Y, Li Z, Han F. Electroacupuncture for patients with irritable bowel syndrome: a systematic review and meta-analysis protocol. Medicine 97(31), e11627 (2018).

5. Papatheodoridis GV, Vlachogiannakos J, Karaitianos I, Karamanolis DG. A Greek survey of community prevalence and characteristics of constipation. Eur. J. Gastroenterol. Hepatol. 22(3), 354-360 (2010).

6. Fosnes GS, Lydersen S, Farup PG. Constipation and diarrhoea - common adverse drug reactions? A cross sectional study in the general population. BMC. Clin. Pharmacol. 11, 2 (2011).

7. Wald A, Mueller-Lissner S, Kamm MA et al. Survey of laxative use by adults with self-defined constipation in South America and Asia: a comparison of six countries. Alimentary pharmacology \& therapeutics. 31(2), 274-284 (2010).

8. Suares NC, Ford AC. Prevalence of, and risk factors for, chronic idiopathic constipation in the community: systematic review and meta-analysis. Am. J. Gastroenterol. 106(9), 1582-1591 (2011).

9. Preston SH, Stokes A. Sources of population aging in more and less developed countries. Popul. Dev. Rev. 38(2), 221-236 (2012).

10. Xue J, Li J, Liang J, Chen S. The prevalence of mild cognitive impairment in China: a systematic review. Aging. Dis. 9(4), 706-715 (2018).

11. Ning XH. Hospice and palliative care in mainland China: history, current status and challenges. Chin. Med. Sci. J. 33(4), 199-203 (2018).

12. Chelimsky G, Kovacic K, Simpson P et al. Benign joint hypermobility minimally impacts autonomic abnormalities in pediatric subjects with chronic functional pain disorders. J. Pediatr. 177, 49-52 (2016).

13. Lynch SV, Pedersen O. The human intestinal microbiome in health and disease. New Engl. J. Med. 375(24), 2369-2379 (2016).

14. Liu R, Hong J, Xu X et al. Gut microbiome and serum metabolome alterations in obesity and after weight-loss intervention. Nat. Med. 23(7), 859-868 (2017).

15. Ruohtula T, De Goffau MC, Nieminen JK et al. Maturation of gut microbiota and circulating regulatory T cells and development of IgE sensitization in early life. Front. Immunol. 10, 2494 (2019). 
16. Lemessurier KS, Iverson AR, Chang TC et al. Allergic inflammation alters the lung microbiome and hinders synergistic co-infection with H1N1 influenza virus and Streptococcus pneumoniae in C57BL/6 mice. Sci. Rep. 9(1), 19360 (2019).

17. Li X, Yuan FL. Comment on "Gut microbiota as a driver of inflammation in nonalcoholic fatty liver disease". Mediat. inflamm. 2018,3746509 (2018).

18. Ferrucci L, Fabbri E. Inflammageing: chronic inflammation in ageing, cardiovascular disease, and frailty. Nat. Rev. Cardiol. 15(9), 505-522 (2018).

19. Parthasarathy G, Chen J, Chen X et al. Relationship between microbiota of the colonic mucosa vs feces and symptoms, colonic transit, and methane production in female patients with chronic constipation. Gastroenterology 150(2), 367-379 e361 (2016).

20. Zhu L, Liu W, Alkhouri R et al. Structural changes in the gut microbiome of constipated patients. Physiol. Genomics. 46(18), 679-686 (2014).

21. Quigley EM. The enteric microbiota in the pathogenesis and management of constipation. Best Pract. Res. Clin. Gastroenterol. 25(1), 119-126 (2011).

22. Tap J, Derrien M, Tornblom H et al. Identification of an intestinal microbiota signature associated with severity of irritable bowel syndrome. Gastroenterology 152(1), 111-123 e118 (2017).

23. Chassard C, Dapoigny M, Scott KP et al. Functional dysbiosis within the gut microbiota of patients with constipated-irritable bowel syndrome. Aliment. Pharm. Ther. 35(7), 828-838 (2012).

24. Caporaso JG, Kuczynski J, Stombaugh J et al. QIIME allows analysis of high-throughput community sequencing data. Nat. Methods. 7(5), 335-336 (2010).

25. Desantis TZ, Hugenholtz P, Larsen N et al. Greengenes, a chimera-checked $16 \mathrm{~S}$ rRNA gene database and workbench compatible with ARB. Appl. Environ. Microbiol. 72(7), 5069-5072 (2006).

26. Gill SR, Pop M, Deboy RT et al. Metagenomic analysis of the human distal gut microbiome. Science 312(5778), 1355-1359 (2006).

27. Altschul SF, Madden TL, Schaffer AA et al. Gapped BLAST and PSI-BLAST: a new generation of protein database search programs. Nucleic Acids Res. 25(17), 3389-3402 (1997).

28. Lozupone CA, Hamady M, Kelley ST, Knight R. Quantitative and qualitative beta diversity measures lead to different insights into factors that structure microbial communities. Appl. Environ. Microbiol. 73(5), 1576-1585 (2007).

29. Paliy O, Shankar V. Application of multivariate statistical techniques in microbial ecology. Mol. Ecol. 25(5), 1032-1057 (2016).

30. Buttigieg PL, Ramette A. A guide to statistical analysis in microbial ecology: a community-focused, living review of multivariate data analyses. FEMS Microbiol. Ecol. 90(3), 543-550 (2014).

31. Segata N, Izard J, Waldron L et al. Metagenomic biomarker discovery and explanation. Genome Biol. 12(6), R60 (2011).

32. Chen Y, Yang F, Lu H et al. Characterization of fecal microbial communities in patients with liver cirrhosis. Hepatology 54(2), 562-572 (2011).

33. Levy EI, Lemmens R, Vandenplas Y, Devreker T. Functional constipation in children: challenges and solutions. Pediatr. Health Med. Ther. 8, 19-27 (2017).

34. Shin JE, Park KS, Nam K. [Chronic functional constipation]. Korean J. Gastroenterol. 73(2), 92-98 (2019).

35. Sharma A, Rao S. Constipation: pathophysiology and current therapeutic approaches. Handb. Exp. Pharmacol. 239, 59-74 (2017).

36. Rao SS, Rattanakovit K, Patcharatrakul T. Diagnosis and management of chronic constipation in adults. Nat. Rev. Gastroenterol. Hepatol. 13(5), 295-305 (2016).

37. De Meij TG, De Groot EF, Eck A et al. Characterization of microbiota in children with chronic functional constipation. PloS ONE 11(10), e0164731 (2016).

38. Huang L, Jiang $\mathrm{H}$, Zhu M et al. Prevalence and risk factors of chronic constipation among women aged 50 years and older in Shanghai, China. Med. Sci. Monit. 23, 2660-2667 (2017).

39. Cryan JF, Dinan TG. Mind-altering microorganisms: the impact of the gut microbiota on brain and behaviour. Nat. Rev. Neurosci. 13(10), 701-712 (2012).

40. Moloney RD, Desbonnet L, Clarke G, Dinan TG, Cryan JF. The microbiome: stress, health and disease. Mamm. Genome 25(1-2), 49-74 (2014).

41. Halmos T, Suba I. [Physiological patterns of intestinal microbiota. The role of dysbacteriosis in obesity, insulin resistance, diabetes and metabolic syndrome]. Orv. Hetil. 157(1), 13-22 (2016).

42. He C, Shan Y, Song W. Targeting gut microbiota as a possible therapy for diabetes. Nutr. Res. 35(5), 361-367 (2015).

43. Esteve E, Ricart W, Fernandez-Real JM. Gut microbiota interactions with obesity, insulin resistance and type 2 diabetes: did gut microbiote co-evolve with insulin resistance? Curr. Opin. Clin. Nutr. Metab. Care. 14(5), 483-490 (2011).

44. Hartstra AV, Bouter KE, Backhed F, Nieuwdorp M. Insights into the role of the microbiome in obesity and Type 2 diabetes. Diabetes Care 38(1), 159-165 (2015). 
45. Jeffery IB, O’Toole PW, Ohman L et al. An irritable bowel syndrome subtype defined by species-specific alterations in faecal microbiota. Gut 61(7), 997-1006 (2012).

46. Ohara T. Identification of the microbial diversity after fecal microbiota transplantation therapy for chronic intractable constipation using 16s rRNA amplicon sequencing. PloS ONE. 14(3), e0214085 (2019).

47. Li Q, Wu T, Liu R, Zhang M, Wang R. Soluble dietary fiber reduces trimethylamine metabolism via gut microbiota and co-regulates host AMPK pathways. Mol. Nutr. Food Res. 61(12), (2017).

48. Wu GD, Chen J, Hoffmann C et al. Linking long-term dietary patterns with gut microbial enterotypes. Science 334(6052), 105-108 (2011).

49. Kurokawa S, Kishimoto T, Mizuno $\mathrm{S}$ et al. The effect of fecal microbiota transplantation on psychiatric symptoms among patients with irritable bowel syndrome, functional diarrhea and functional constipation: an open-label observational study. J. Affect. Disord. 235, 506-512 (2018).

50. Mancabelli L, Milani C, Lugli GA et al. Unveiling the gut microbiota composition and functionality associated with constipation through metagenomic analyses. Sci. Rep. 7(1), 9879 (2017).

51. Kang DW, Dibaise JK, Ilhan ZE et al. Gut microbial and short-chain fatty acid profiles in adults with chronic constipation before and after treatment with lubiprostone. Anaerobe. 33, 33-41 (2015).

52. Strati F, Cavalieri D, Albanese D et al. New evidences on the altered gut microbiota in autism spectrum disorders. Microbiome. 5(1), 24 (2017).

53. Wolf PG, Parthasarathy G, Chen J et al. Assessing the colonic microbiome, hydrogenogenic and hydrogenotrophic genes, transit and breath methane in constipation. Neurogastroenterol. Motil. 29(10), 1-9 (2017).

54. Louis P, Flint HJ. Formation of propionate and butyrate by the human colonic microbiota. Environ. Microbiol. 19(1), 29-41 (2017).

55. Tazoe H, Otomo Y, Kaji I, Tanaka R, Karaki SI, Kuwahara A. Roles of short-chain fatty acids receptors, GPR 41 and GPR 43 on colonic functions. J. Physiol. Pharmacol. 59(Suppl. 2), 251-262 (2008).

56. Bach Knudsen KE, Laerke HN, Hedemann MS et al. Impact of diet-modulated butyrate production on intestinal barrier function and inflammation. Nutrients 10(10), E1499 (2018).

57. Spiller R. Review article: probiotics and prebiotics in irritable bowel syndrome. Aliment. Pharm. Ther. 28(4), 385-396 (2008).

58. Moro-Velazquez L, Gomez-Garcia JA, Godino-Llorente JI. Corrigendum: voice pathology detection using modulation spectrum-optimized metrics. Front. Bioeng. Biotechnol. 4, 67 (2016).

59. Curro D, Ianiro G, Pecere S, Bibbo S, Cammarota G. Probiotics, fibre and herbal medicinal products for functional and inflammatory bowel disorders. Br. J. Pharmacol. 174(11), 1426-1449 (2017).

60. Ford AC, Harris LA, Lacy BE, Quigley EMM, Moayyedi P. Systematic review with meta-analysis: the efficacy of prebiotics, probiotics, synbiotics and antibiotics in irritable bowel syndrome. Aliment. Pharm. Ther. 48(10), 1044-1060 (2018).

61. Zhang Y, Li L, Guo C et al. Effects of probiotic type, dose and treatment duration on irritable bowel syndrome diagnosed by Rome III criteria: a meta-analysis. BMC Gastroenterol. 16(1), 62 (2016). 
